\title{
DISTANCE SETS OF TWO SUBSETS OF VECTOR SPACES OVER FINITE FIELDS
}

\author{
DOOWON KOH AND HAE-SANG SUN
}

(Communicated by Alexander Iosevich)

\begin{abstract}
We investigate the size of the distance set determined by two subsets of finite dimensional vector spaces over finite fields. A lower bound of the size is given explicitly in terms of cardinalities of the two subsets. As a result, we improve upon the results by Rainer Dietmann. In the case that one of the subsets is a product set, we obtain further improvement on the estimate.
\end{abstract}

\section{INTRODUCTION}

Let $E, F$ be finite subsets of $\mathbb{R}^{d}$. The distance set determined by $E$ and $F$ is defined by

$$
\Delta(E, F)=\{|x-y|: x \in E, y \in F\},
$$

where $|\cdot|$ denotes the standard norm on $\mathbb{R}^{d}$. Much attention has been paid to estimating the cardinality of the distance set $\Delta(E, F)$. In the case of $E=F$, Erdös [4] first addressed this problem and showed that

$$
|\Delta(E, E)| \gg|E|^{\frac{1}{d}}
$$

where $|E|$ denotes the number of elements in $E$. Here, and throughout the paper, we use $\gg$ conventionally. That is to say, there exists a $c>0$ such that $\Delta(E, E)>c|E|^{\frac{1}{d}}$ for all $E$. Taking the set $E$ as a piece of the integer lattice, the Erdös distance conjecture says that for every $\varepsilon>0$, there exists a $c_{\varepsilon}>0$ such that

$$
|\Delta(E, E)| \geq c_{\varepsilon}|E|^{\frac{2}{d}-\varepsilon} .
$$

In dimension two, the conjecture has recently been solved by Guth and Katz [5], who proved that

$$
|\Delta(E, E)| \gg \frac{|E|}{\log |E|} .
$$

However, the Erdős distance conjecture is still open for higher dimensions. See [12], 16], 17], and the references contained therein for recent developments on the Erdős distance problem in higher dimensions.

As an analog of the Euclidean Erdős distance problem, Bourgain, Katz, and Tao [1] posed and studied the finite field version of the Erdös distance problem in two dimensions. The Erdős distance problem in the finite field setting has been recently

Received by the editors April 20, 2013.

2010 Mathematics Subject Classification. Primary 52C10, 11T23.

Key words and phrases. Erdős distance problem, finite fields.

The first and second authors were supported by the Basic Science Research Program through the National Research Foundation of Korea (NRF) funded by the Ministry of Education, Science and Technology (2012R1A1A1001510, 2010-0023248). 
studied by various researchers (see [2], 6], [10, [1], [18, [19, and [20]). Let $\mathbb{F}_{q}^{d}$ be a $d$-dimensional vector space over the finite field $\mathbb{F}_{q}$ with $q$ elements. We shall always assume that the characteristic of $\mathbb{F}_{q}$ is greater than two. In the finite field setting, given two sets $E, F \subset \mathbb{F}_{q}^{d}$, the distance set is defined similarly by

$$
\Delta(E, F)=\left\{\|x-y\| \in \mathbb{F}_{q}: x \in E, y \in F\right\},
$$

where $\|\cdot\|$ is defined by $\|m\|=m_{1}^{2}+\cdots+m_{d}^{2}$ for $m=\left(m_{1}, \ldots, m_{d}\right) \in \mathbb{F}_{q}^{d}$. Here, observe that the function $\|\cdot\|$ on $\mathbb{F}_{q}^{d}$ is not a norm, but the value is invariant under the rotations in $\mathbb{F}_{q}^{d}$.

Assuming that $E \subset \mathbb{F}_{p}^{2}$ with a prime $p \equiv 3(\bmod 4)$, the aforementioned authors [1] proved that if $|E| \leq p^{2-\varepsilon}$ for some $\varepsilon>0$, then there exists a $\delta(\varepsilon)>0$ such that $|\Delta(E, E)| \gg|E|^{\frac{1}{2}+\delta(\varepsilon)}$. However, the value $\delta(\varepsilon)$ was not given in an explicit form. Furthermore, this result cannot be obtained for general finite fields, because one may take $E=\mathbb{F}_{p} \times \mathbb{F}_{p}$ for the prime subfield $\mathbb{F}_{p}$ of $\mathbb{F}_{q}$.

For general fields $\mathbb{F}_{q}$, Iosevich and Rudnev [9] obtained results on lower bounds with explicit exponents for the size of distance sets for $\mathbb{F}_{q}^{d}, d \geq 2$. More precisely, they proved that if $E \subset \mathbb{F}_{q}^{d}$ and $|E| \gg q^{\frac{d}{2}}$, then

$$
|\Delta(E, E)| \gg \min \left\{q, \frac{|E|}{q^{\frac{d-1}{2}}}\right\} .
$$

In [15], Shparlinski derived an explicit lower bound of the number of the distances between arbitrary two sets: If $E, F \subset \mathbb{F}_{q}^{d}$, then

$$
|\Delta(E, F)|>\frac{|E||F| q}{q^{d+1}+|E||F|} \geq \frac{1}{2} \min \left\{q, \frac{|E||F|}{q^{d}}\right\} .
$$

Dietmann [3] recently obtained a new lower bound for $|\Delta(E, F)|$. In fact, he proved that if $E, F \subset \mathbb{F}_{q}^{d},|F| \geq|E|$, and $|E||F| \geq(900+\log q) q^{d}$, then

$$
|\Delta(E, F)| \gg \begin{cases}\min \left\{q, \frac{|F|}{q^{\frac{d-1}{2}} \log q}\right\} & \text { for } d \geq 2, \\ \min \left\{q, \frac{|E|^{\frac{1}{2}}|F|}{q \log q}\right\} & \text { for } d=2 .\end{cases}
$$

In order to obtain an estimate on the average of a product of two spherical sums, he made use of the pigeonhole principle, which is a main reason for the presence of the $\log q$ factor in (1.2). One might make a naive speculation that explicit Fourier analysis instead of the pigeonhole principle could remove the $\log q$ factor.

Another point worth noting is that both results of Shparlinski and Dietmann are nontrivial only if

$$
|E||F|>q^{d}
$$

Here notice that the condition (1.3) is optimal for even $d$. In fact, if $d \geq 2$ is even and $i^{2}=-1$ for some $i \in \mathbb{F}_{q}$, then setting $E=F$ and

$$
E=\left\{\left(t_{1}, i t_{1}, \ldots, t_{j}, i t_{j}, \ldots, t_{d / 2}, i t_{d / 2}\right) \in \mathbb{F}_{q}^{d}: t_{j} \in \mathbb{F}_{q}, j=1, \ldots, d / 2\right\},
$$

it can be easily shown that $|E||F|=q^{d}$ and $|\Delta(E, F)|=1$. On the other hand, absence of such an example for odd $d$ leads us to speculate that (1.3) might be relaxed further. 
There are three aims of the present paper. The first two of them are responses to the previous speculations, which are consequences of the main theorems in Section 3. First of all, we shall observe that Dietmann's result can be improved so that the $\log q$ factor can be eliminated in (1.2). In fact, in Corollary 3.6, we have : If $|E||F| \gg q^{d}$ and $|E| \leq|F|$, then we have

$$
|\Delta(E, F)| \gg \begin{cases}\min \left\{q, \frac{|F|}{\left.q^{\frac{d-1}{2}}\right\}}\right. & \text { if } d \geq 2, \\ \min \left\{q, \frac{|E|^{\frac{1}{2}}|F|}{q}\right\} & \text { if } d=2 .\end{cases}
$$

Secondly, we show that in certain cases, a condition milder than (1.3) assures nontriviality of distance sets. For example, in Theorem 3.3. we show that if $d \geq 3$ is odd and $1 \leq|E|<q^{\frac{d-1}{2}}$, then

$$
|\Delta(E, F)| \geq \min \left\{\frac{q}{2}, \frac{|E||F|}{8 q^{d-1}}\right\}
$$

and, therefore, $|\Delta(E, F)|>1$ if $|E||F|>8 q^{d-1}$. Finally, we also show in Theorem 3.8 that if one of the two subsets of $\mathbb{F}_{q}$ is a product set, then a much stronger lower bound for the size of the distance set is obtained.

For precise statements and more explanations, please refer to the theorems and remarks in Section 3 ,

\section{Discrete Fourier analysis}

Iosevich and Rudnev [9] adapted the discrete Fourier analysis to measure the size of distance sets in the finite field setting. As a result, they developed a powerful machinery for deriving results on the Erdös distance problem. In this section, we review it and collect estimates on several quantities, namely the Fourier transform of spheres, counting function of points with a given distance, and spherical sums, which are involved in the lower bound for the distance set.

We begin with the definition of the Fourier transform. Given a function $f: \mathbb{F}_{q}^{d} \rightarrow$ $\mathbb{C}$, the Fourier transform of $f$ is given by the form

$$
\widehat{f}(m)=\frac{1}{q^{d}} \sum_{x \in \mathbb{F}_{q}^{d}} \chi(-m \cdot x) f(x) \quad \text { for } m \in \mathbb{F}_{q}^{d} .
$$

Here, and throughout this paper, we denote by $\chi$ a fixed nontrivial additive character of $\mathbb{F}_{q}$. It can be easily checked that the results on the distance problems are independent of the choice of the character. Recall that the orthogonality relation of $\chi$ says that

$$
\sum_{x \in \mathbb{F}_{q}^{d}} \chi(m \cdot x)= \begin{cases}0 & \text { if } m \neq(0, \ldots, 0), \\ q^{d} & \text { if } m=(0, \ldots, 0) .\end{cases}
$$

The following Fourier inversion formula follows immediately from a direct application of the orthogonality relation of $\chi$ :

$$
f(x)=\sum_{m \in \mathbb{F}_{q}^{d}} \chi(m \cdot x) \widehat{f}(m) .
$$


The discrete version of Plancherel's theorem says that

$$
\sum_{m \in \mathbb{F}_{q}^{d}}|\widehat{f}(m)|^{2}=q^{-d} \sum_{x \in \mathbb{F}_{q}^{d}}|f(x)|^{2} .
$$

From now on, by abuse of notation, we identify the symbol $E$ for a subset $E \subset \mathbb{F}_{q}^{d}$ with the characteristic function $\chi_{E}$ on $E$. Then the Plancherel theorem for $E$ is interpreted as

$$
\sum_{m \in \mathbb{F}_{q}^{d}}|\widehat{E}(m)|^{2}=q^{-d} \sum_{x \in \mathbb{F}_{q}^{d}}|E(x)|^{2}=q^{-d}|E| .
$$

Let us denote by $G, K, S$ the Gauss sum, Kloosterman sum, and Salié sum, respectively. In other words, for $a, b \in \mathbb{F}_{q}^{*}$, let us set

$$
G=\sum_{s \in \mathbb{F}_{q}^{*}} \eta(s) \chi(s), \quad K=\sum_{s \in \mathbb{F}_{q}^{*}} \chi\left(a s+b s^{-1}\right), \quad \text { and } \quad S=\sum_{s \in \mathbb{F}_{q}^{*}} \eta(s) \chi\left(a s+b s^{-1}\right),
$$

where $\eta$ denotes the quadratic character of $\mathbb{F}_{q}^{*}:=\mathbb{F}_{q} \backslash\{0\}$. It is well known that they satisfy

$$
|G|=\sqrt{q},|K| \leq 2 \sqrt{q} \text {, and }|S| \leq 2 \sqrt{q} .
$$

For proofs of estimates on these exponential sums, see [13, p. 193] and [8, pp. 322323].

For each $t \in \mathbb{F}_{q}$, we define a sphere with radius $t$ as the set

$$
S_{t}=\left\{x \in \mathbb{F}_{q}^{d}:\|x\|=t\right\} .
$$

The Fourier transform on $S_{t}$ is closely related to the aforementioned exponential sums. It was proved in [7] that for $t \in \mathbb{F}_{q}, m \in \mathbb{F}_{q}^{d}$,

$$
\widehat{S}_{t}(m)=q^{-1} \delta_{0}(m)+q^{-d-1} \eta^{d}(-1) G^{d} \sum_{r \in \mathbb{F}_{q}^{*}} \eta^{d}(r) \chi\left(t r+\frac{\|m\|}{4 r}\right),
$$

where $\delta_{0}(m)=1$ if $m=(0, \ldots, 0)$ and $\delta_{0}(m)=0$ otherwise. Given two sets $E, F \subset \mathbb{F}_{q}^{d}$, we consider a counting function $\nu: \mathbb{F}_{q} \rightarrow \mathbb{N} \cup\{0\}$ defined by

$$
\nu(t)=|\{(x, y) \in E \times F:\|x-y\|=t\}| \quad \text { for } t \in \mathbb{F}_{q} .
$$

For $E \subset \mathbb{F}_{q}^{d}$, let us set

$$
\mathfrak{M}(E):=\max _{r \in \mathbb{F}_{q}} \sum_{m \in S_{r}}|\widehat{E}(m)|^{2} \text {, and } \mathfrak{M}^{*}(E):=\max _{r \in \mathbb{F}_{q}^{*}} \sum_{m \in S_{r}}|\widehat{E}(m)|^{2} .
$$

Note that $\sum_{m \in S_{r}}|\widehat{E}(m)|^{2}$ is a finite analog of spherical average $\int_{S^{d-1}}|\widehat{\mu}(t \omega)|^{2} d \omega$ in the classical Falconer distance problem. Here $S^{d-1}$ is a $(d-1)$-dimensional sphere and $\mu$ is a Borel measure. Refer to 9 for the details.

The three quantities $\widehat{S}_{t}, \nu(t)$, and $\mathfrak{M}(E)$ are closely related by the following lemma, (2.6), and (2.7).

Lemma 2.1. We have

$$
\nu(t)=q^{2 d} \sum_{m \in \mathbb{F}_{q}^{d}} \widehat{S}_{t}(m) \widehat{E}(m) \widehat{F}(m) .
$$


Proof. This can be checked by applying the Fourier inversion formula to $S_{t}(x-y)$. For each $t \in \mathbb{F}_{q}$, we have

$$
\nu(t)=\sum_{x \in E, y \in F} S_{t}(x-y)=\sum_{x, y \in \mathbb{F}_{q}^{d}} E(x) F(y) \sum_{m \in \mathbb{F}_{q}^{d}} \chi(m \cdot(x-y)) \widehat{S}_{t}(m) .
$$

Then (2.3) follows from the definition of the Fourier transform.

Now in the following subsections we collect estimates and expressions for the three quantities, which are necessary to prove the main theorems in Section 3 .

2.1. Fourier transform of $S_{t}$. Clearly by definition we have

$$
\widehat{S}_{t}(0, \ldots, 0)=q^{-d}\left|S_{t}\right| \leq 2 q^{-1}
$$

for $t \in \mathbb{F}_{q}$. We also have

Proposition 2.2. (1) For $m \neq(0,0, \cdots, 0)$,

$$
\left|\widehat{S}_{t}(m)\right| \leq\left\{\begin{array}{cl}
q^{-\frac{d}{2}} & \text { if } d: \text { even, } t=0, \text { and }\|m\|=0, \\
2 q^{-\frac{d+1}{2}} & \text { otherwise. }
\end{array}\right.
$$

In particular, $\left|\widehat{S}_{t}(m)\right| \leq 2 q^{-\frac{d+1}{2}}$ for all $t \in \mathbb{F}_{q}$ if $d$ is odd, and for all $t \neq 0$ if $d$ is even.

(2) For $m, m^{\prime} \in \mathbb{F}_{q}^{d}$, we have

$$
\sum_{t \in \mathbb{F}_{q}} \widehat{S}_{t}(m) \widehat{\widehat{S}}_{t}\left(m^{\prime}\right)=q^{-1} \delta_{0}(m) \delta_{0}\left(m^{\prime}\right)+q^{-d-1} \sum_{s \in \mathbb{F}_{q}^{*}} \chi\left(s\left(\|m\|-\left\|m^{\prime}\right\|\right)\right) .
$$

Proof. Observe that for any $d \geq 2$, we have

$$
\left|\sum_{r \in \mathbb{F}_{q}^{*}} \eta^{d}(r) \chi\left(t r+\frac{\|m\|}{4 r}\right)\right| \leq 2 q^{\frac{1}{2}}
$$

unless $d$ is even, $t=0$, and $\|m\|=0$. Statement (1) is an immediate consequence from (2.1) and (2.2).

For $m \in \mathbb{F}_{q}^{d}, t \in \mathbb{F}_{q}$, write $\widehat{S}_{t}(m)=q^{-1} \delta_{0}(m)+R_{t}(m)$, where $R_{t}(m)$ denotes the second term of the right-hand side in (2.2). It follows that for $m, m^{\prime} \in \mathbb{F}_{q}^{d}$,

$$
\begin{aligned}
\sum_{t \in \mathbb{F}_{q}} \widehat{S}_{t}(m) \overline{\widehat{S}_{t}}\left(m^{\prime}\right)=\sum_{t \in \mathbb{F}_{q}} q^{-2} \delta_{0}(m) \delta_{0}\left(m^{\prime}\right)+q^{-1} \delta_{0}(m) \sum_{t \in \mathbb{F}_{q}} \overline{R_{t}}\left(m^{\prime}\right) \\
+q^{-1} \delta_{0}\left(m^{\prime}\right) \sum_{t \in \mathbb{F}_{q}} R_{t}(m)+\sum_{t \in \mathbb{F}_{q}} R_{t}(m) \overline{R_{t}}\left(m^{\prime}\right) .
\end{aligned}
$$

By the orthogonality relation for $\chi$, the sums in the second and third terms in (2.5) vanish. Thus, (2.5) is equal to

$$
\begin{aligned}
& q^{-1} \delta_{0}(m) \delta_{0}\left(m^{\prime}\right)+q^{-d-2} \sum_{s, s^{\prime} \in \mathbb{F}_{q}^{*}} \eta^{d}(s) \bar{\eta}^{d}\left(s^{\prime}\right) \chi\left(\frac{\|m\|}{4 s}-\frac{\left\|m^{\prime}\right\|}{4 s^{\prime}}\right) \sum_{t \in \mathbb{F}_{q}} \chi\left(\left(s-s^{\prime}\right) t\right) \\
& =q^{-1} \delta_{0}(m) \delta_{0}\left(m^{\prime}\right)+q^{-d-1} \sum_{s \in \mathbb{F}_{q}^{*}} \chi\left(\frac{\|m\|-\left\|m^{\prime}\right\|}{4 s}\right) .
\end{aligned}
$$

Then statement (2) follows from an observation that the change of variables, $\frac{1}{4 s} \mapsto s$, is a permutation on $\mathbb{F}_{q}^{*}$. 
2.2. Counting function $\nu(t)$. We investigate some properties of $\nu(t)$. The $l^{2}$ estimate of the counting function $\nu$ takes the following form.

Proposition 2.3. For $E, F \subset \mathbb{F}_{q}^{d}$, we have

$$
\begin{aligned}
& \sum_{t \in \mathbb{F}_{q}} \nu^{2}(t) \leq q^{-1}|E|^{2}|F|^{2}+q^{2 d}|F| \mathfrak{M}(E), \text { and } \\
& \sum_{t \in \mathbb{F}_{q}} \nu^{2}(t) \leq q^{-1}|E|^{2}|F|^{2}+q^{3 d}\left|\sum_{m \in S_{0}} \widehat{\widehat{E}}(m) \widehat{F}(m)\right|^{2}+q^{2 d}|F| \mathfrak{M}^{*}(E) .
\end{aligned}
$$

Proof. Squaring both sides of (2.3) and summing over $t \in \mathbb{F}_{q}$, we have

$$
\sum_{t \in \mathbb{F}_{q}^{d}} \nu^{2}(t)=q^{4 d} \sum_{m, m^{\prime} \in \mathbb{F}_{q}^{d}} \overline{\widehat{E}}(m) \widehat{F}(m) \widehat{E}\left(m^{\prime}\right) \overline{\widehat{F}}\left(m^{\prime}\right) \sum_{t \in \mathbb{F}_{q}} \widehat{S}_{t}(m) \overline{\widehat{S}_{t}}\left(m^{\prime}\right) .
$$

Since $\mathbb{F}_{q}^{*}=\mathbb{F}_{q} \backslash\{0\}$, it follows from Proposition 2.2 that (2.8) is equal to

$$
\begin{aligned}
\frac{|E|^{2}|F|^{2}}{q} & +q^{3 d-1} \sum_{m, m^{\prime} \in \mathbb{F}_{q}^{d}} \overline{\widehat{E}}(m) \widehat{F}(m) \widehat{E}\left(m^{\prime}\right) \overline{\widehat{F}}\left(m^{\prime}\right) \sum_{s \in \mathbb{F}_{q}} \chi\left(s\left(\|m\|-\left\|m^{\prime}\right\|\right)\right) \\
& -q^{3 d-1}\left|\sum_{m \in \mathbb{F}_{q}^{d}} \widehat{\widehat{E}}(m) \widehat{F}(m)\right|^{2} .
\end{aligned}
$$

Since the last term in (2.9) is negative, applying the orthogonality relation for $\chi$, we obtain that

$$
\sum_{t \in \mathbb{F}_{q}^{d}} \nu^{2}(t) \leq \frac{|E|^{2}|F|^{2}}{q}+q^{3 d} \sum_{\|m\|=\left\|m^{\prime}\right\|} \overline{\widehat{E}}(m) \widehat{F}(m) \widehat{E}\left(m^{\prime}\right) \widehat{\widehat{F}}\left(m^{\prime}\right),
$$

where the last summation is over $m, m^{\prime} \in \mathbb{F}_{q}^{d}$ with $\|m\|=\left\|m^{\prime}\right\|$. This can be rewritten as

$$
\sum_{t \in \mathbb{F}_{q}^{d}} \nu^{2}(t) \leq \frac{|E|^{2}|F|^{2}}{q}+q^{3 d} \sum_{r \in \mathbb{F}_{q}}\left|\sum_{m \in S_{r}} \bar{E}(m) \widehat{F}(m)\right|^{2} .
$$

Observe that

$$
\begin{aligned}
\sum_{r \in \mathbb{F}_{q}}\left|\sum_{m \in S_{r}} \widehat{\widehat{E}}(m) \widehat{F}(m)\right|^{2} & \leq \sum_{r \in \mathbb{F}_{q}}\left(\sum_{m \in S_{r}}|\widehat{E}(m)|^{2}\right)\left(\sum_{m \in S_{r}}|\widehat{F}(m)|^{2}\right) \\
& \leq \mathfrak{M}(E) \sum_{m \in \mathbb{F}_{q}^{d}}|\widehat{F}(m)|^{2}=q^{-d} \mathfrak{M}(E)|F| .
\end{aligned}
$$

Then (2.6) follows from this inequality.

Note that (2.11) is still valid even when " $r \in \mathbb{F}_{q}$ " and $\mathfrak{M}(E)$ are replaced by " $r \in \mathbb{F}_{q}^{*}$ " and $\mathfrak{M}^{*}(E)$. Since $\mathbb{F}_{q}=\mathbb{F}_{q}^{*} \cup\{0\}$, the inequality (2.7) is easily derived from (2.10) and the variant of (2.11).

In the next few paragraphs, we collect a number of lemmas on $\nu(0)$, which are going to be utilized for the proof of Theorem 3.5 
Proposition 2.4. Suppose that $d \geq 2$ is even and $E, F \subset \mathbb{F}_{q}^{d}$ with $|E \| F| \geq 16 q^{d}$. Then we have

$$
\begin{aligned}
& (|E||F|-\nu(0))^{2} \geq \frac{|E|^{2}|F|^{2}}{36}, \text { and } \\
& q^{3 d}\left|\sum_{m \in S_{0}} \overline{\widehat{E}}(m) \widehat{F}(m)\right|^{2}-\nu^{2}(0) \leq q^{-1}|E|^{2}|F|^{2} .
\end{aligned}
$$

Proof. Since $d \geq 2$ is even, it follows from (2.2) and (2.3) that

$$
\begin{aligned}
\nu(0) & =q^{2 d} \sum_{m \in \mathbb{F}_{q}^{d}} \overline{\widehat{E}}(m) \widehat{F}(m)\left(q^{-1} \delta_{0}(m)+q^{-d-1} G^{d} \sum_{r \in \mathbb{F}_{q}^{*}} \chi\left(\frac{\|m\|}{4 r}\right)\right) \\
& =q^{-1}|E \| F|+q^{d-1} G^{d} \sum_{m \in \mathbb{F}_{q}^{d}} \overline{\widehat{E}}(m) \widehat{F}(m)\left(\sum_{s \in \mathbb{F}_{q}} \chi(s\|m\|)-1\right) \\
& =q^{-1}|E \| F|+q^{d} G^{d} \sum_{m \in S_{0}} \overline{\widehat{E}}(m) \widehat{F}(m)-q^{d-1} G^{d} \sum_{m \in \mathbb{F}_{q}^{d}} \overline{\widehat{E}}(m) \widehat{F}(m) .
\end{aligned}
$$

Notice that from (2.12) we obtain

$$
|\nu(0)| \leq q^{-1}|E||F|+2 q^{d}|G|^{d} \sum_{m \in \mathbb{F}_{q}^{d}}|\widehat{E}(m)||\widehat{F}(m)| .
$$

From the Cauchy-Schwarz inequality and the Plancherel theorem, we have

$$
\sum_{m \in \mathbb{F}_{q}^{d}}|\bar{E}(m)||\widehat{F}(m)| \leq q^{-d}|E|^{\frac{1}{2}}|F|^{\frac{1}{2}}
$$

and, therefore,

$$
|\nu(0)| \leq q^{-1}|E||F|+2 q^{\frac{d}{2}}|E|^{\frac{1}{2}}|F|^{\frac{1}{2}} .
$$

This inequality implies that

$$
|E||F|-\nu(0) \geq|E||F|-|\nu(0)| \geq\left(1-q^{-1}\right)|E||F|-2 q^{\frac{d}{2}}|E|^{\frac{1}{2}}|F|^{\frac{1}{2}} .
$$

Since $q \geq 3$ and $|E||F| \geq 16 q^{d}$, we see that

$$
|E||F|-\nu(0) \geq \frac{2|E||F|}{3}-2 q^{\frac{d}{2}}|E|^{\frac{1}{2}}|F|^{\frac{1}{2}} \geq \frac{|E||F|}{6} \geq 0 .
$$

The statement follows immediately from this observation.

For the second statement, let us set

$$
M(E, F)=q^{-1}|E||F|-q^{d-1} G^{d} \sum_{m \in \mathbb{F}_{q}^{d}} \widehat{\widehat{E}}(m) \widehat{F}(m)
$$

for the convenience of calculations. Plugging (2.12) into $\nu^{2}(0)=\nu(0) \overline{\nu(0)}$ and expanding it, we see that

$$
\begin{aligned}
\nu^{2}(0)= & q^{3 d}\left|\sum_{m \in S_{0}} \overline{\widehat{E}}(m) \widehat{F}(m)\right|^{2}+q^{d} G^{d} \overline{M(E, F)} \sum_{m \in S_{0}} \overline{\widehat{E}}(m) \widehat{F}(m) \\
& +q^{d} \overline{G^{d}} M(E, F) \sum_{m \in S_{0}} \widehat{E}(m) \overline{\widehat{F}}(m)+|M(E, F)|^{2}
\end{aligned}
$$


Since $\nu^{2}(0)$ is a nonnegative integer and $|M(E, F)|^{2} \geq 0$, the equality above with $|G|=q^{\frac{1}{2}}$ implies that

$$
q^{3 d}\left|\sum_{m \in S_{0}} \overline{\widehat{E}}(m) \widehat{F}(m)\right|^{2}-\nu^{2}(0) \leq 2 q^{\frac{3 d}{2}}|M(E, F)| \sum_{m \in \mathbb{F}_{q}^{d}}|\widehat{E}(m)||\widehat{F}(m)| .
$$

Note that the second factor is bounded by $q^{-d}|E|^{\frac{1}{2}}|F|^{\frac{1}{2}}$ using the Cauchy-Schwarz inequality and the Plancherel theorem. Using this estimate and the definition of $M(E, F)$, it is easy to see that

$$
|M(E, F)| \leq q^{-1}|E||F|+q^{d-1}\left|G^{d}\right| \sum_{m \in \mathbb{F}_{q}^{d}}|\widehat{E}(m)||\widehat{F}(m)| \leq q^{-1}|E||F|+q^{\frac{d}{2}-1}|E|^{\frac{1}{2}}|F|^{\frac{1}{2}} .
$$

Putting all estimates above together, we obtain

$$
q^{3 d}\left|\sum_{m \in S_{0}} \widehat{\widehat{E}}(m) \widehat{F}(m)\right|^{2}-\nu^{2}(0) \leq 2 q^{\frac{d}{2}-1}|E|^{\frac{3}{2}}|F|^{\frac{3}{2}}+2 q^{d-1}|E||F| .
$$

A direct computation shows that if $|E||F| \geq 16 q^{d}$, then the R.H.S. of the previous inequality is less than or equal to $4 q^{\frac{d}{2}-1}|E|^{\frac{3}{2}}|F|^{\frac{3}{2}} \leq q^{-1}|E|^{2}|F|^{2}$. This completes the proof.

2.3. Spherical sums $\mathfrak{M}(E)$ and $\mathfrak{M}^{*}(E)$. The following lemma plays a crucial role in proving results in the case of dimension two. The proof can be found in Chapman et al. [2, Lemma 4.4].

Lemma 2.5. If $E \subset \mathbb{F}_{q}^{2}$, then one has

$$
\mathfrak{M}^{*}(E) \leq \sqrt{3} q^{-3}|E|^{\frac{3}{2}} .
$$

For higher dimensions, we need:

Proposition 2.6. For odd $d \geq 3$, we have

$$
\mathfrak{M}(E) \leq \min \left\{q^{-d}|E|, 2 q^{-d-1}|E|+2 q^{-\frac{3 d+1}{2}}|E|^{2}\right\} .
$$

For even $d \geq 2$, we have

$$
\mathfrak{M}^{*}(E) \leq \min \left\{q^{-d}|E|, 2 q^{-d-1}|E|+2 q^{-\frac{3 d+1}{2}}|E|^{2}\right\} .
$$

Proof. For each $r \in \mathbb{F}_{q}$, the Plancherel theorem yields $\sum_{m \in S_{r}}|\widehat{E}(m)|^{2} \leq \sum_{m \in \mathbb{F}_{q}^{d}}|\widehat{E}(m)|^{2}$ $=q^{-d}|E|$. Hence we obtain that

$$
\mathfrak{M}(E) \leq q^{-d}|E| .
$$

From the definition of the Fourier transform, it follows that for each $r \in \mathbb{F}_{q}$,

$$
\begin{aligned}
\sum_{m \in S_{r}}|\widehat{E}(m)|^{2} & =q^{-d} \sum_{x, y \in E} \widehat{S_{r}}(x-y) \\
& =q^{-d}|E| \widehat{S_{r}}(0, \ldots, 0)+q^{-d} \sum_{x, y \in E: x \neq y} \widehat{S_{r}}(x-y) .
\end{aligned}
$$


For odd $d \geq 3$, we see from (2.4) and Proposition 2.2 (1) that

$$
\mathfrak{M}(E) \leq 2 q^{-d-1}|E|+2 q^{-\frac{3 d+1}{2}}|E|^{2} .
$$

Combining with (2.13), this estimate yields the first statement.

Finally, arguments similar to the above give the same upper bound for $\mathfrak{M}^{*}(E)$.

When $E$ is of a special form, namely a product set, we have a stronger bound.

Proposition 2.7. Let $E=\underline{E} \times A \subset \mathbb{F}_{q}^{d-1} \times \mathbb{F}_{q}$. Then $\mathfrak{M}(E) \leq 2 q^{-d-1}|A|^{2}|\underline{E}|$. In particular, if $E=A^{d}$ for a subset $A$ of $\mathbb{F}_{q}$, then $\mathfrak{M}(E) \leq 2 q^{-d-1}|E|^{1+\frac{1}{d}}$.

Proof. We see from the definition of the Fourier transform that for $m=\left(\underline{m}, m_{d}\right) \in$ $\mathbb{F}_{q}^{d-1} \times \mathbb{F}_{q}$,

$$
\widehat{E}(m)=\widehat{E} \times A\left(\underline{m}, m_{d}\right)=\underline{\widehat{E}}(\underline{m}) \widehat{A}\left(m_{d}\right),
$$

where $\underline{\widehat{E}}(\underline{m})=q^{-(d-1)} \sum_{\underline{x} \in \mathbb{F}_{q}^{d-1}} \chi(-\underline{m} \cdot \underline{x}) \underline{E}(\underline{x})$ and $\widehat{A}\left(m_{d}\right)=q^{-1} \sum_{s \in \mathbb{F}_{q}} \chi\left(-s \cdot m_{d}\right) A(s)$.

Then, for each $r \in \mathbb{F}_{q}$, we can write

$$
\sum_{m \in S_{r}}|\widehat{E}(m)|^{2}=\sum_{\underline{m} \in \mathbb{F}_{q}^{d-1}}|\underline{\widehat{E}}(\underline{m})|^{2}\left(\sum_{m_{d} \in \mathbb{F}_{q}: m_{d}^{2}=r-\|\underline{m}\|}\left|\widehat{A}\left(m_{d}\right)\right|^{2}\right) .
$$

Since $\left|\widehat{A}\left(m_{d}\right)\right| \leq|\widehat{A}(0)|=q^{-1}|A|$ for all $m_{d} \in \mathbb{F}_{q}$, and $\left|\left\{m_{d} \in \mathbb{F}_{q}: m_{d}^{2}=r-\|\underline{m}\|\right\}\right| \leq$ 2 for each $r \in \mathbb{F}_{q}, \underline{m} \in \mathbb{F}_{q}^{d-1}$, we see that

$$
\sum_{m \in S_{r}}|\widehat{E}(m)|^{2} \leq 2 q^{-2}|A|^{2} \sum_{\underline{m} \in \mathbb{F}_{q}^{d-1}}|\underline{\widehat{E}}(\underline{m})|^{2}=2 q^{-d-1}|A|^{2}|\underline{E}|,
$$

where the last equality follows from the Plancherel theorem in dimension $(d-1)$.

Let $E=A \times \cdots \times A=\underline{E} \times A \subset \mathbb{F}_{q}^{d-1} \times \mathbb{F}_{q}$. Then the second assertion follows from the observation that $|A|^{2}|\underline{E}|=|E|^{1+1 / d}$.

\section{Distance SEts: Main Results}

Now, we review standard distance formulas which were originally due to Iosevich and Rudnev 9 .

Basic inequalities the distance function enjoys are as follows.

Lemma 3.1. Let $E, F \subset \mathbb{F}_{q}^{d}, d \geq 2$. Then we have

$$
\begin{aligned}
& |\Delta(E, F)| \geq \frac{|E|^{2}|F|^{2}}{\sum_{t \in \mathbb{F}_{q}} \nu^{2}(t)} \text { and } \\
& |\Delta(E, F)| \geq \frac{(|E||F|-\nu(0))^{2}}{\sum_{t \in \mathbb{F}_{q}^{*}} \nu^{2}(t)} .
\end{aligned}
$$

Proof. Since $|E||F|=\sum_{t \in \mathbb{F}_{q}} \nu(t)$ and $|E||F|-\nu(0)=\sum_{t \in \mathbb{F}_{q}^{*}} \nu(t)$, the CauchySchwarz inequality yields

$$
|E|^{2}|F|^{2}=\left(\sum_{t \in \mathbb{F}_{q}} \nu(t)\right)^{2} \leq|\Delta(E, F)| \sum_{t \in \mathbb{F}_{q}} \nu^{2}(t)
$$


and

$$
(|E||F|-\nu(0))^{2}=\left(\sum_{t \in \mathbb{F}_{q}^{*}} \nu(t)\right)^{2} \leq|\Delta(E, F)| \sum_{t \in \mathbb{F}_{q}^{*}} \nu^{2}(t) .
$$

Thus, (3.1) and (3.2) follow immediately from these observations.

Remark 3.2. As we shall see, inequality (3.1) is used to prove our distance results in odd dimensions. On the other hand, the inequality (3.2) is useful in the even dimensional case. Iosevich and Rudnev [9] and Dietmann [3] made use of formula (3.2) to derive distance results. Consequently, they obtained the nontrivial distance results in the case when $|E||F| \gg q^{d}$. In this paper we want to point out that if the dimension $d \geq 3$ is odd, then formula (3.1) enables us to yield nontrivial results whenever $|E \|| F \mid \gg q^{d-1}$. In [3, Dietmann obtained the result in (1.2) by estimating $\sum_{t \in \mathbb{F}_{q}^{*}} \nu^{2}(t)$. To the end, he applied the pigeonhole principle so that his result contains the $\log q$ factor. However, our main results below show that the $\log q$ factor can be removed.

Theorem 3.3. If $d \geq 3$ is odd, and $E, F \subset \mathbb{F}_{q}^{d}$, then

$$
|\Delta(E, F)| \geq \begin{cases}\min \left\{\frac{q}{2}, \frac{|E||F|}{8 q^{d-1}}\right\} & \text { if } 1 \leq|E|<q^{\frac{d-1}{2}}, \\ \min \left\{\frac{q}{2}, \frac{|F|}{\left.8 q^{\frac{d-1}{2}}\right\}}\right. & \text { if } q^{\frac{d-1}{2}} \leq|E|<q^{\frac{d+1}{2}}, \\ \min \left\{\frac{q}{2}, \frac{|E||F|}{2 q^{d}}\right\} & \text { if } q^{\frac{d+1}{2}} \leq|E| \leq q^{d} .\end{cases}
$$

Proof. Combining (3.1) with (2.6), we see that

$$
|\Delta(E, F)| \geq \frac{|E|^{2}|F|^{2}}{q^{-1}|E|^{2}|F|^{2}+q^{2 d}|F| \mathfrak{M}(E)} .
$$

Since $d$ is odd, from Proposition 2.6, we have

$$
\mathfrak{M}(E) \leq \begin{cases}4 q^{-d-1}|E| & \text { if } 1 \leq|E|<q^{\frac{d-1}{2}} \\ 4 q^{-\frac{3 d+1}{2}}|E|^{2} & \text { if } q^{\frac{d-1}{2}} \leq|E|<q^{\frac{d+1}{2}} \\ q^{-d}|E| & \text { if } q^{\frac{d+1}{2}} \leq|E| \leq q^{d} .\end{cases}
$$

After combining (3.3) with this estimate, a direct computation enables us to finish the proof of Theorem 3.3 .

Remark 3.4. It is not hard to see that Theorem 3.3 is stronger than Dietmann's result (1.2) for $d \geq 3$ odd (see Corollary 3.6 below). In addition, notice that Theorem 3.3 improves Shparlinski's result (1.1) in the case when $d \geq 3$ is odd and $1 \leq|E|<q^{(d+1) / 2}$. One important point is that if $|E||F| \leq q^{d}$, then Shparlinski's result says nothing more than $|\Delta(E, F)| \geq 1$. The same thing can be said for Dietmann's result, because his result depends on a strong assumption that $|E||F| \geq(900+\log q) q^{d}$. In contrast, Theorem 3.3 gives meaningful information about $|\Delta(E, F)|$ whenever $d \geq 3$ is odd and $8 q^{d-1}<|E||F| \leq q^{d}$. For example, if $d \geq 3$ is odd, $|E|=q^{(d-1) / 2}-1$, and $|F|=q^{(d+1) / 2}$, then $|E||F|=q^{d}-q^{(d+1) / 2}<q^{d}$, but $|\Delta(E, F)| \geq q / 12$.

Now, we state and prove our main theorem for even dimensions, which improves Dietmann's result (1.2) (see also Corollary [3.6 below). 
Theorem 3.5. (1) Let $d \geq 2$ be even and $E, F \subset \mathbb{F}_{q}^{d}$. If $|E||F| \geq 16 q^{d}$, then we have

$$
|\Delta(E, F)| \geq \begin{cases}\frac{q}{144} & \text { for } 1 \leq|E|<q^{\frac{d-1}{2}}, \\ \frac{1}{144} \min \left\{q, \frac{|F|}{\left.2 q^{\frac{d-1}{2}}\right\}}\right. & \text { for } q^{\frac{d-1}{2}} \leq|E|<q^{\frac{d+1}{2}} \\ \frac{1}{144} \min \left\{q, \frac{2|E||F|}{q^{d}}\right\} & \text { for } q^{\frac{d+1}{2}} \leq|E| \leq q^{d} .\end{cases}
$$

(2) In addition, if $d=2$ and $|E||F| \geq 16 q^{2}$, then

$$
|\Delta(E, F)| \geq \frac{1}{72} \min \left\{\frac{q}{2}, \frac{|E|^{\frac{1}{2}}|F|}{\sqrt{3} q}\right\} .
$$

Furthermore, if $d=2$ and $-1 \notin \mathbb{F}_{q}^{* 2}$, then

$$
|\Delta(E, F)| \geq \min \left\{\frac{q}{2}, \frac{|E|^{\frac{1}{2}}|F|}{2(\sqrt{3}+1) q}\right\} .
$$

Proof. Note that (2.7) can be rewritten as

$$
\sum_{t \in \mathbb{F}_{q}^{*}} \nu^{2}(t) \leq q^{-1}|E|^{2}|F|^{2}+q^{2 d}|F| \mathfrak{M}^{*}(E)+q^{3 d}\left|\sum_{m \in S_{0}} \overline{\widehat{E}}(m) \widehat{F}(m)\right|^{2}-\nu(0)^{2} .
$$

Applying this inequality with ones in Proposition 2.4 to (3.2), we obtain that

$$
|\Delta(E, F)| \geq \frac{|E|^{2}|F|^{2} / 36}{2 q^{-1}|E|^{2}|F|^{2}+q^{2 d}|F| \mathfrak{M}^{*}(E)} .
$$

Now, observe that Proposition 2.6 implies

$$
\mathfrak{M}^{*}(E) \leq \begin{cases}4 q^{-d-1}|E| & \text { if } 1 \leq|E|<q^{\frac{d-1}{2}} \\ 4 q^{-\frac{3 d+1}{2}}|E|^{2} & \text { if } q^{\frac{d-1}{2}} \leq|E|<q^{\frac{d+1}{2}} \\ q^{-d}|E| & \text { if } q^{\frac{d+1}{2}} \leq|E| \leq q^{d}\end{cases}
$$

Combining this inequality with (3.4) and considering the dominant term in terms of $|E|$, we obtain from a direct calculation that

$$
|\Delta(E, F)| \geq \begin{cases}\frac{1}{144} \min \left\{q, \frac{|E||F|}{2 q^{d-1}}\right\} & \text { for } 1 \leq|E|<q^{\frac{d-1}{2}}, \\ \frac{1}{144} \min \left\{q, \frac{|F|}{\left.2 q^{\frac{d-1}{2}}\right\}}\right. & \text { for } q^{\frac{d-1}{2}} \leq|E|<q^{\frac{d+1}{2}}, \\ \frac{1}{144} \min \left\{q, \frac{2|E||F|}{q^{d}}\right\} & \text { for } q^{\frac{d+1}{2}} \leq|E| \leq q^{d} .\end{cases}
$$

Since $|E||F| \geq 16 q^{d}$, we conclude the first part of the theorem.

For statement (2), suppose that $E, F \subset \mathbb{F}_{q}^{2}$ with $|E||F| \geq 16 q^{2}$. Applying Lemma 2.5 to inequality (3.4), we conclude that

$$
|\Delta(E, F)| \geq \frac{|E|^{2}|F|^{2}}{36\left(2 q^{-1}|E|^{2}|F|^{2}+\sqrt{3} q|E|^{\frac{3}{2}}|F|\right)} \geq \frac{1}{72} \min \left\{\frac{q}{2}, \frac{|E|^{\frac{1}{2}}|F|}{\sqrt{3} q}\right\}
$$

and complete the proof. 
For the last statement, let $E, F \subset \mathbb{F}_{q}^{2}$. In addition, assume that $-1 \in \mathbb{F}_{q}$ is not a square. Then $S_{0}=\{(0,0)\}$. Therefore, it follows from (2.7) in Proposition 2.3 that

$$
\sum_{t \in \mathbb{F}_{q}} \nu^{2}(t) \leq q^{-1}|E|^{2}|F|^{2}+q^{6}|\widehat{\widehat{E}}(0,0) \widehat{F}(0,0)|^{2}+q^{4}|F| \mathfrak{M}^{*}(E) .
$$

Since $\bar{E}(0,0)=q^{-2}|E|$ and $\widehat{F}(0,0)=q^{-2}|F|$, an application of Lemma 2.5 yields that

$$
\sum_{t \in \mathbb{F}_{q}} \nu^{2}(t) \leq q^{-1}|E|^{2}|F|^{2}+q^{-2}|E|^{2}|F|^{2}+\sqrt{3} q|E|^{\frac{3}{2}}|F| .
$$

Now, observe that $q^{-2}|E|^{2}|F|^{2} \leq q|E|^{\frac{3}{2}}|F|$ for $E, F \subset \mathbb{F}_{q}^{2}$. It therefore follows that

$$
\sum_{t \in \mathbb{F}_{q}} \nu^{2}(t) \leq q^{-1}|E|^{2}|F|^{2}+(1+\sqrt{3}) q|E|^{\frac{3}{2}}|F| .
$$

By this inequality and (3.1) in Lemma 3.1, we see that

$$
|\Delta(E, F)| \geq \frac{|E|^{2}|F|^{2}}{q^{-1}|E|^{2}|F|^{2}+(1+\sqrt{3}) q|E|^{\frac{3}{2}}|F|} \geq \min \left\{\frac{q}{2}, \frac{|E|^{\frac{1}{2}}|F|}{2(\sqrt{3}+1) q}\right\} .
$$

Thus, the proof is complete.

Putting Theorems 3.3 and 3.5 together, we show:

Corollary 3.6. If $|E||F| \gg q^{d}$ and $|E| \leq|F|$, then we have

$$
|\Delta(E, F)| \gg \begin{cases}\min \left\{q, \frac{|F|}{q^{\frac{d-1}{2}}}\right\} & \text { if } d \geq 2, \\ \min \left\{q, \frac{|E|^{\frac{1}{2}}|F|}{q}\right\} & \text { if } d=2 .\end{cases}
$$

Proof. First, let $d \geq 3$ be odd. Due to the hypothesis, it suffices to consider only the first condition in Theorem 3.3. In this case, we have $\min \left\{q / 2,|E||F| / 8 q^{d-1}\right\} \gg q$. Note also that $|F| / q^{(d-1) / 2} \gg q$ since $|F| \gg q^{d} /|E| \geq q^{(d+1) / 2}$. From similar arguments with Theorem 3.5, we are also able to verify the corollary for even $d \geq 2$. The case of $d=2$ follows immediately from Theorem 3.5 . Hence the proof is finished.

Remark 3.7. In dimension two, the second part of Theorem 3.5 enables us to improve the first part of Theorem [3.5] in the case when $q \leq|E| \leq q^{2}$. As mentioned above, if $d \geq 3$ is odd, then $|\Delta(E, F)|>1$ whenever $8 q^{d-1}<|E||F| \leq q^{d}$. However, this is not true any more in even dimensions as observed in example (1.4). For this reason, we need the assumption that $|E||F| \geq 16 q^{d}$ in Theorem 3.5. The last part of Theorem 3.5 says that if we assume that $d=2$ and $-1 \in \mathbb{F}_{q}$ is not a square, then we can drop the assumption in the second part of Theorem 3.5 that $|E||F| \geq 16 q^{2}$. In this case, we have nontrivial distance results whenever $|E|^{\frac{1}{2}}|F|>2(\sqrt{3}+1) q$.

The following result can be obtained by finding a good upper bound of $\mathfrak{M}(E)$ for any product set $E$ in $\mathbb{F}_{q}^{d}$. 
Theorem 3.8. Let $d \geq 2$. Suppose that $E=A \times A \times \cdots \times A \subset \mathbb{F}_{q}^{d}$ is a product set and $F \subset \mathbb{F}_{q}^{d}$. Then we have

$$
|\Delta(E, F)| \geq \min \left\{\frac{q}{2}, \frac{|E|^{1-\frac{1}{d}}|F|}{4 q^{d-1}}\right\} .
$$

Proof. Let $d \geq 2$. Suppose that $E=A \times A \times \cdots \times A \subset \mathbb{F}_{q}^{d}$ is a product set and $F \subset \mathbb{F}_{q}^{d}$. Combining (3.1) with (2.6), we have

$$
|\Delta(E, F)| \geq \frac{|E|^{2}|F|^{2}}{q^{-1}|E|^{2}|F|^{2}+q^{2 d}|F| \mathfrak{M}(E)} .
$$

From Proposition 2.7 we have

$$
\mathfrak{M}(E) \leq 2 q^{-d-1}|E|^{1+\frac{1}{d}} .
$$

Applying this inequality to (3.6) gives

$$
|\Delta(E, F)| \geq \frac{|E|^{2}|F|^{2}}{q^{-1}|E|^{2}|F|^{2}+2 q^{d-1}|E|^{1+\frac{1}{d}}|F|} \geq \min \left\{\frac{q}{2}, \frac{|E|^{1-\frac{1}{d}}|F|}{4 q^{d-1}}\right\} .
$$

This completes the proof.

Remark 3.9. Notice that if $q^{d / 2} \leq|E| \leq q^{d} / 2^{d}$, then the conclusion of Theorem 3.8 is superior to the conclusions of both Theorems 3.3 and 3.5. In particular, the conclusion of Theorem 3.8 holds true without the assumption of Theorem 3.5 that $|E||F| \geq 16 q^{d}$. In fact, it yields nontrivial results whenever $|E|^{1-1 / d}|F|>4 q^{d-1}$, a weaker condition than $|E||F| \geq 16 q^{d}$.

Remark 3.10. As shown in the proofs of Proposition 2.7 and Theorem 3.8, the assumption that $E=A \times A \times \cdots \times A$ can be replaced by a weaker condition that $E=\underline{E} \times A \subset \mathbb{F}_{q}^{d-1} \times \mathbb{F}_{q}$ and $|\underline{E}|=|A|^{d-1}$. In particular, if we assume that $E=\underline{E} \times A \subset \mathbb{F}_{q}^{d-1} \times \mathbb{F}_{q}$ and $|\underline{E}|>|A|^{d-1}$, then we could obtain a much stronger conclusion than (3.5).

\section{REFERENCES}

[1] J. Bourgain, N. Katz, and T. Tao, A sum-product estimate in finite fields, and applications, Geom. Funct. Anal. 14 (2004), no. 1, 27-57, DOI 10.1007/s00039-004-0451-1. MR2053599 (2005d:11028)

[2] Jeremy Chapman, M. Burak Erdoğan, Derrick Hart, Alex Iosevich, and Doowon Koh, Pinned distance sets, $k$-simplices, Wolff's exponent in finite fields and sum-product estimates, Math. Z. 271 (2012), no. 1-2, 63-93, DOI 10.1007/s00209-011-0852-4. MR2917133

[3] R. Dietmann, On the Erdös-Falconer distance problem for two sets of different size in vector spaces over finite fields, accepted for publication in Monatsh. Math.

[4] P. Erdös, On sets of distances of $n$ points, Amer. Math. Monthly 53 (1946), 248-250. $\operatorname{MR} 0015796(7,471 \mathrm{c})$

[5] L. Guth and N. Katz, On the Erdös distinct distance problem in the plane, preprint.

[6] Alex Iosevich and Doowon Koh, The Erdös-Falconer distance problem, exponential sums, and Fourier analytic approach to incidence theorems in vector spaces over finite fields, SIAM J. Discrete Math. 23 (2008/09), no. 1, 123-135, DOI 10.1137/060669875. MR2452874 (2009i:52011)

[7] Alex Iosevich and Doowon Koh, Extension theorems for spheres in the finite field setting, Forum Math. 22 (2010), no. 3, 457-483, DOI 10.1515/FORUM.2010.025. MR2652707 (2011e:42010) 
[8] Henryk Iwaniec and Emmanuel Kowalski, Analytic number theory, American Mathematical Society Colloquium Publications, vol. 53, American Mathematical Society, Providence, RI, 2004. MR2061214 (2005h:11005)

[9] A. Iosevich and M. Rudnev, Erdös distance problem in vector spaces over finite fields, Trans. Amer. Math. Soc. 359 (2007), no. 12, 6127-6142 (electronic), DOI 10.1090/S0002-9947-0704265-1. MR2336319 (2008k:11130)

[10] Doowon Koh and Chun-Yen Shen, Sharp extension theorems and Falconer distance problems for algebraic curves in two dimensional vector spaces over finite fields, Rev. Mat. Iberoam. 28 (2012), no. 1, 157-178, DOI 10.4171/RMI/672. MR2904136

[11] Doowon Koh and Chun-Yen Shen, The generalized Erdös-Falconer distance problems in vector spaces over finite fields, J. Number Theory 132 (2012), no. 11, 2455-2473, DOI 10.1016/j.jnt.2012.05.003. MR2954983

[12] Nets Hawk Katz and Gábor Tardos, A new entropy inequality for the Erdös distance problem, Towards a theory of geometric graphs, Contemp. Math., vol. 342, Amer. Math. Soc., Providence, RI, 2004, pp. 119-126, DOI 10.1090/conm/342/06136. MR2065258(2005f:52033)

[13] Rudolf Lidl and Harald Niederreiter, Finite fields, With a foreword by P. M. Cohn, 2nd ed., Encyclopedia of Mathematics and its Applications, vol. 20, Cambridge University Press, Cambridge, 1997. MR1429394 (97i:11115)

[14] Hans Salié, Über die Kloostermanschen Summen $S(u, v ; q)$ (German), Math. Z. 34 (1932), no. 1, 91-109, DOI 10.1007/BF01180579. MR1545243

[15] Igor E. Shparlinski, On the set of distances between two sets over finite fields, Int. J. Math. Math. Sci., posted on 2006, Art. ID 59482, 5, DOI 10.1155/IJMMS/2006/59482. MR2251747 (2007f:12006)

[16] József Solymosi and Van Vu, Distinct distances in high dimensional homogeneous sets, Towards a theory of geometric graphs, Contemp. Math., vol. 342, Amer. Math. Soc., Providence, RI, 2004, pp. 259-268, DOI 10.1090/conm/342/06146. MR2065269(2005m:52026)

[17] József Solymosi and Van H. Vu, Near optimal bounds for the Erdös distinct distances problem in high dimensions, Combinatorica 28 (2008), no. 1, 113-125, DOI 10.1007/s00493-008-20991. MR2399013 (2009f:52042)

[18] Le Anh Vinh, Explicit Ramsey graphs and Erdös distance problems over finite Euclidean and non-Euclidean spaces, Electron. J. Combin. 15 (2008), no. 1, Research Paper 5, 18. MR2368910(2008m:05150)

[19] Le Anh Vinh, The Erdös-Falconer distance problem on the unit sphere in vector spaces over finite fields, SIAM J. Discrete Math. 25 (2011), no. 2, 681-684, DOI 10.1137/080736545. MR2817524 (2012f:05153)

[20] Van H. Vu, Sum-product estimates via directed expanders, Math. Res. Lett. 15 (2008), no. 2, 375-388, DOI 10.4310/MRL.2008.v15.n2.a14. MR2385648(2009e:11023)

Department of Mathematics, Chungbuk National University, Cheonguu, Chungbuk 361-763, Republic OF KoreA

E-mail address: koh131@chungbuk.ac.kr

Department of Mathematics, Chungbuk National University, Cheonguu, Chungbuk 361-763, RePublic of KoreA

E-mail address: haesang@chungbuk.ac.kr

Current address: Department of Mathematical Sciences, Ulsan National Institute of Science and Technology, UNIST-gil 50, Ulsan 689-798, Republic of Korea

E-mail address: haesang.sun@gmail.com 